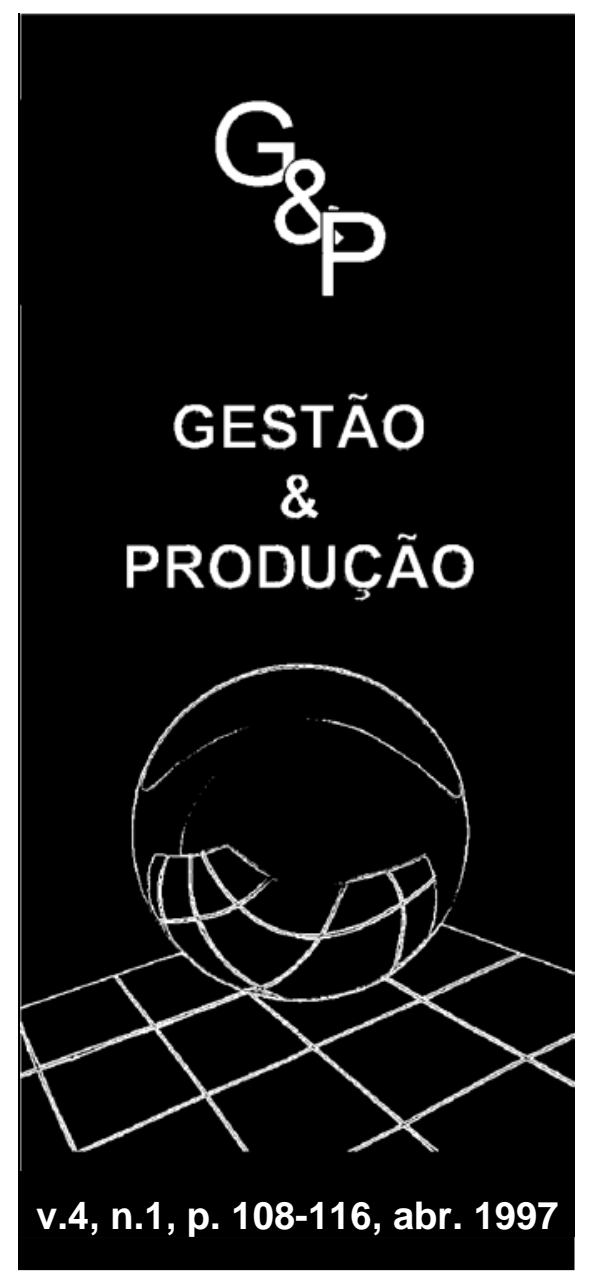

\title{
SISTEMATIZAÇÃO PARA A IMPLANTA- ÇÃO INTEGRADA DE SISTEMAS DE PLANEJAMENTO FINO DA PRODUÇÃO
}

\author{
Eng. Fábio Favaretto, MSC \\ Dep. de Eng. Mecânica da Escola de Eng. de São Carlos - USP \\ Av. Dr. Carlos Botelho, 1465 \\ e-mail: favareto@tigre-prod.prod.eesc.sc.usp.br
}

Prof. Dr. Carlos Frederico Bremer

Área de Eng. de Produção do Dep. de Eng. Mecânica da Escola de Eng. de São Carlos - USP

Av. Dr. Carlos Botelho, 1465

\section{Resumo}

As mudanças ocorridas no cenário mundial têm levado as empresas de manufatura a buscar um aumento da sua flexibilidade. O Planejamento e Controle da Produção (PCP) é a função da empresa que é mais sensível às mudanças necessárias em direção a um aumento da flexibilidade. Os sistemas de Planejamento Fino da Produção (PFP) são ferramentas que surgiram com o objetivo de auxiliar a função de PCP nessa busca pela flexibilidade. Os sistemas de PFP fazem a ligação entre a dinâmica do chão-de-fábrica e o ambiente de planejamento, e a sua implantação é um fator crítico para que as necessidades e expectativas das empresas sejam atendidas. O sistema de PFP necessita de integrações com outros sistemas de apoio a funções da manufatura. O objetivo deste trabalho é propor uma sistematização para a implantação integrada de sistemas de PFP. Esta proposta foi aplicada em um ambiente simulado de manufatura, chamado Fábrica Integrada Modelo (FIM). Na FIM esta proposta foi testada e validada.

\section{Palavras-chave: sistemas de planejamento fino da produção, implantação.}

\section{Introdução}

A

globalização da economia tem provocado grandes mudanças no cenário mundial, as quais afetam diretamente as empresas de manufatura. A concorrência encontra-se acirrada em diversos setores industriais. Para uma empresa manter-se competitiva nesse mercado, é necessário possuir vantagens 
competitivas. Estas podem ser alcançadas de diversas maneiras, uma das quais é o aumento da flexibilidade da empresa.

A busca da flexibilidade acarreta mudanças estruturais e organizacionais nas empresas de manufatura. Essas mudanças são sentidas em diversos setores da empresa, mas um dos mais afetados é o setor de planejamento e controle da produção (PCP).

O setor de PCP processa uma grande quantidade de dados, provenientes de diversos setores da empresa. Para o processamento eficaz e rápido desses dados, existem sistemas de informação que fazem o processamento de todos, ou de parte dos dados do setor de PCP. Esses sistemas são responsáveis pela automação de diversas tarefas do PCP. Para que esses sistemas atendam às necessidades e expectativas da empresa, é necessário que estejam integrados entre si.
Uma deficiência percebida em empresas de manufatura em face das novas condições do mercado é a ligação entre o planejamento e o controle da produção. Isso ocorre porque o planejamento da produção não trabalha próximo à dinâmica do chão-de-fábrica. $\mathrm{Na}$ tentativa de solução dessa deficiência, foram desenvolvidos os sistemas "Leitstand" (MELLO, 1994). Esses sistemas fazem um refinamento do planejamento da produção, além de trabalharem com o retorno das informações do chão-de-fábrica, permitindo o controle da produção e correções no planejamento. Assim, um sistema de PFP não elimina o processo de PCP tradicional, mas sim o complementa, permitindo interferir de maneira visual na programação dos recursos produtivos.

Independente das características do sistema, a sua implantação é um fator crítico para o êxito de sua utilização (ROZENFELD \& FAVARETTO, 1993).

\section{Objetivo do Trabalho}

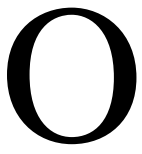

objetivo principal deste trabalho é desenvolver uma sistemática de auxílio às empresas de manufatura para a implantação integrada de sistemas de planejamento fino da produção. Esta sistemática tem o objetivo de orientar as atividades e decisões envolvidas na implantação.

\section{Sistematização da Implantação Integrada de Sistemas de PFP}

\subsection{Arquitetura}

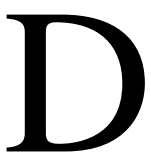
entre algumas arquiteturas pesquisadas, aquela que apresentou maior aderência aos requisitos deste trabalho foi a Arquitetura Geral de Integração - AGIR (BREMER, 1995).

A Arquitetura Geral de Integração fornece a estrutura de uma metodologia de planejamento e implantação da manufatura integrada por computador (CIM). Para isso, é necessário que seja adotada uma forma de representação que permita a visualização de cada uma das partes de uma empresa e seus inter-relacionamentos. A notação adotada para representação da AGIR, por ser a mais apropriada, foi a do paradigma de orientação a objetos. As principais classes envolvidas são:

- modelo de empresa e métodos;

- projeto de integração;

- organização e estratégia

- componentes CIM.

Os objetivos da AGIR são os seguintes:

- representar todos os objetos, serviços e relacionamentos envolvidos no processo 
de planejamento e implantação da manufatura integrada por computador;

- promover sinergia e entendimento das atividades da empresa e

- garantir continuamente a integração.

A adoção da AGIR deve-se principalmente ao fato de prever a forma de planejamento da implantação. Essa utilização é um dos objetivos deste trabalho. A forma de planejamento da implantação define o direcionamento a ser dado em relação à estratégia de implantação da integração.

As formas de planejamento da implantação previstas na AGIR permitem que seja definida a estratégia de integração de sistemas. Essa estratégia reflete a experiência da empresa em projetos relacionados com automação e processamento de informações, a formação das pessoas envolvidas, e o direcionamento estratégico da empresa, entre outros.

A AGIR propõe três formas de planejamento da implantação, que serão detalhadas a seguir.

A implantação integrada de sistemas de PFP utilizando a primeira forma é caracterizada pela utilização de sistemas isolados e pela falta de relacionamento com a estratégia de negócios da empresa. Isto porque as estratégias de negócio da empresa definem as funções que devem ser priorizadas para que a empresa mantenha ou alcance uma boa participação no mercado consumidor.

Neste caso, o sistema de PFP é implantado isoladamente, independentemente de haver em funcionamento outros sistemas que poderiam ser integrados, como um sistema de MRP ou de coleta de dados de chão-de-fábrica. Este tipo de implantação ocorre nos casos em que o setor de programação da empresa decide isoladamente a aquisição de um sistema de PFP, sem preocupações estratégicas nem de integração com outros sistemas.

A implantação integrada de sistemas de PFP, utilizando a segunda forma de planejamento da implantação, é caracterizada pelo relacionamento com a estratégia de negócios da empresa, porém o modelo da empresa não é utilizado. Esse modelo é amplo e procura representar todos os processos da empresa. A representação desses processos pode ser dividida em visões, que vislumbram aspectos da empresa. Essas visões são, entre outras: de dados, de processos e de departamentos.

Utilizando a segunda forma, a implantação de sistemas de PFP pode ser feita de maneira integrada com outros sistemas. Esses sistemas podem estar em funcionamento anteriormente ou serem implantados juntamente com o sistema de PFP. A falta de relacionamento com o modelo de negócios da empresa pode provocar problemas com integrações no futuro ou com o fluxo geral de informações da empresa.

A terceira forma de planejamento da implantação é caracterizada por estar relacionada com o modelo de empresa e permitir uma integração contínua e evolutiva. A implantação de sistemas de PFP baseada na terceira forma de planejamento da implantação é aquela que possui melhor chance de atender a todas as expectativas e necessidades da empresa, por ser abrangente e detalhada.

\subsection{Níveis de Integração de Sistemas}

Para este trabalho também serão utilizados os níveis de integração de sistemas propostos por SCHEER (1993). Esses níveis retratam padrões tecnológicos de implantação.

A seguir serão descritos esses níveis. 
A integração organizacional de sistemas independentes consiste na troca de dados entre estes sistemas de maneira não eletrônica. Essa troca de dados pode ser feita de diversas formas, mas principalmente por meio de:

- relatórios impressos e

- comunicação verbal.

A integração de sistemas independentes mediante o uso de ferramentas é caracterizada como sendo o nível 2 de integração entre sistemas. Este tipo de integração é empregado em empresas de manufatura que buscam uma integração rápida e simples entre sistemas.

Neste nível, os sistemas são operados independentemente e a comunicação entre eles ocorre por meio de ferramentas. Por ferramentas entende-se meios auxiliares de comunicação entre sistemas, que podem ser de diversas formas:

- Microcomputadores;

- Consultas e

- Redes.

A transferência de dados entre sistemas é caracterizada como nível 3 de integração entre sistemas. Esse nível de integração tem como principal meio de comunicação os arquivos de transferência. Esses arquivos contêm os dados provenientes de um sistema que devem ser inseridos em outros sistemas. O formato desses dados deve ser compatível com o sistema de destino.

Sistemas compartilhando uma base de dados constituem o nível 4 de integração entre sistemas. Neste tipo de integração, os sistemas são operados independentemente, mas os dados são armazenados em um depósito único.

Contatos de interaplicação via integração de programas constituem o nível mais elevado de integração de sistemas. Neste nível, a integração ocorre no âmbito do código dos sistemas.

Neste nível de integração, um sistema tem acesso direto às transações do outro sistema. Segundo Scheer (SCHEER, 1993), tal tipo de aplicação não pode ser esperado para um futuro próximo. Isso se deve ao fato de que seriam necessários desenvolvimentos em softwares e hardwares ainda não disponíveis em escala comercial.

\subsection{Matrizes de Relações}

A seguir serão propostas duas matrizes que relacionam as formas de planejamento da integração com os níveis de integração de sistemas. Cada posição da matriz será denominada neste trabalho como célula. A referência a cada célula será feita por dois números, dos quais o primeiro representa a forma de planejamento da implantação e o segundo o nível de integração de sistemas.

Uma das matrizes considera a compatibilidade entre as formas de planejamento da implantação e os níveis de integração de sistemas. A outra matriz faz esse relacionamento considerando a adequação da célula para implantação integrada de sistemas de PFP.
Essas matrizes servem de referência para projetos de implantação de sistemas de PFP. Isso porque a escolha da célula reflete a utilização de uma condição específica de implantação, equipamentos e sistemas a serem utilizados. Assim, deve ser escolhida a célula que melhor se adapte às expectativas e condições da empresa. Esta referência deve ser utilizada por empresas que desejem realizar uma implantação integrada de um sistema planejamento fino da produção.

\section{Matriz de compatibilidade}

Esta matriz mostra a compatibilidade entre os níveis de integração de sistemas e as formas de planejamento da implantação. Essa compatibilidade indica se determinado 
nível de integração de sistemas atende às propostas e necessidades de determinada forma de planejamento da implantação.
A representação desta matriz pode ser vista na figura 1.

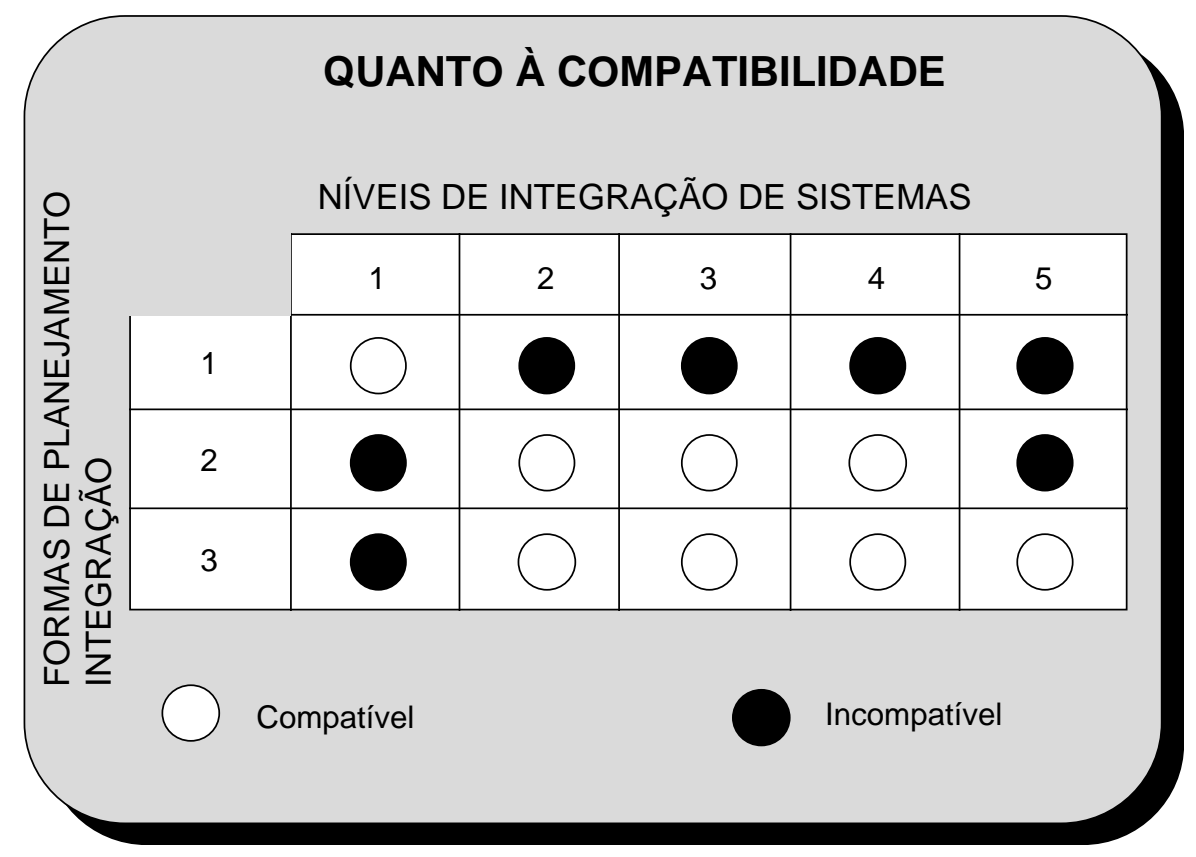

Figura 1 - Matriz de compatibilidade entre formas de planejamento da implantação e níveis de integração de sistemas

Esta matriz representa a possibilidade de utilização conjunta das formas de planejamento e dos níveis de integração de sistemas. Isso porque os níveis mais altos de integração de sistemas necessitam de uma forma de planejamento da integração compatível com eles. A mesma afirmação é válida para os níveis mais baixos. Por exemplo, uma alternativa que considere a forma mais avançada de planejamento da implantação (forma 3) e o nível mais baixo de integração (nível 1) é incompatível, pois este nível não prevê a integração eletrônica entre sistemas.

\section{Matriz de adequação das possibilidades}

Esta matriz permite verificar a adequação de uma determinada alternativa entre formas de planejamento da implantação e níveis de integração de sistemas. Esta matriz mostra se a alternativa apresentada em cada célula é adequada para o caso específico de implantação integrada de sistemas de planejamento fino da produção em empresas de manufatura. Por exemplo, uma alternativa que considere o segundo nível de integração entre sistemas não é adequada para a implantação integrada de sistemas de PFP, pois a integração entre a coleta de dados do chão-de-fábrica e o sistema de PFP necessita de uma freqüente troca de dados, o que não é possível utilizando este nível de integração entre sistemas.

A representação da matriz de adequação das alternativas pode ser vista na figura 2 . 


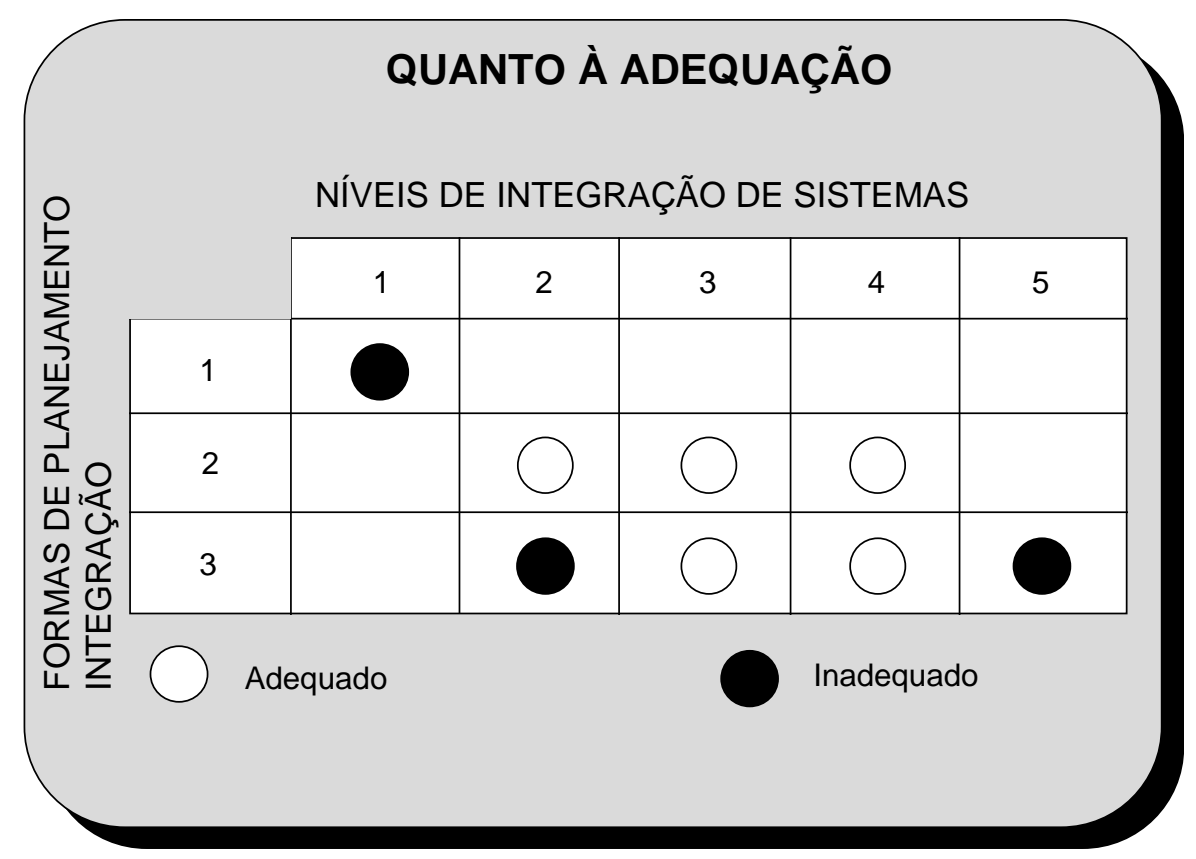

Figura 2 - Matriz de adequação das formas de planejamento da implantação e dos níveis de integração.

Esta matriz faz um refinamento da matriz anterior. Isso porque somente foram incluídas nesta matriz as células consideradas compatíveis na matriz anterior.

\section{Células Estudadas}

A

$\mathrm{s}$ alternativas para um projeto de integração são muitas, porém aquelas que são compatíveis e adequadas são poucas. Este trabalho fará a análise das quatro células que apresentam melhor aderência aos objetivos deste trabalho.

A análise detalhada de todas as alternativas possíveis inviabilizaria o desenvolvimento deste trabalho.

De acordo com uma análise das matrizes apresentadas acima, as células adotadas são as seguintes: 2.3, 2.4, 3.3 e 3.4

A descrição da implantação será feita segundo as etapas da metodologia proposta por ROZENFELD \& FAVARETTO (1993).

A metodologia adotada é dividida em quatro etapas:

- diagnóstico;

- proposição de soluções;

- sistematização e

- implantação.
A seguir, será feita a análise da implantação das células adotadas.

\section{Célula 2.3}

Esta é a célula mais limitada dentre as estudadas. Isso porque tanto a forma de planejamento da implantação como o nível de integração entre sistemas utilizados são limitados.

A integração MRP-PFP apresenta uma limitação quanto à quantidade de dados trocados, devido às limitações do meio físico de transferência (arquivos de transferência). A segunda forma de planejamento da implantação pode limitar esta integração por não observar o modelo de empresa, pois neste estão representados os fluxos de dados. Isso pode levar a um fluxo de dados desordenado. Por exemplo, alguns dados necessários para o PFP podem não ser transmitidos diretamente entre os sistemas, 
ou ser desviados para outro sistema para depois retornar.

Na integração de um sistema de PFP com a coleta de dados esta célula também é limitada, pois a troca de dados pode ser lenta e desordenada. A utilização de arquivos de transferência limita a freqüência de troca de dados entre esses sistemas.

Esta célula é aplicável em empresas nas quais não existe a necessidade de uma comunicação freqüente entre o sistema de PFP e a coleta de dados. Isso ocorre nas empresas em que o tempo de processamento dos componentes do produto é grande ou o ambiente de produção não é complexo. Da mesma forma, esta célula é aplicável nos casos em que o volume de dados trocados entre o sistema de PFP e o sistema MRP não é grande.

A aplicação deste tipo é sugerida para empresas de porte médio, que estão iniciando a integração e a automação de suas funções. Este tipo deve ser usado como uma fase inicial ou preparação para um posterior e mais completo projeto de integração.

\section{Célula 2.4}

Este tipo de integração apresenta uma solução eficiente para a comunicação entre sistemas, porém a forma de planejamento da implantação é limitada.

Na utilização desta célula, as integrações do sistema de PFP são privilegiadas quanto a rapidez, favorecendo a integração PFPColeta de dados. Esta integração é favorecida pois ambos os sistemas compartilham uma base de dados, o que permite que as informações do chão-de-fábrica sejam transferidas automaticamente para os sistemas de PFP.

A integração MRP-PFP nesta célula permite a transferência de uma grande quantidade de dados, porém a falta de relacionamento com o modelo de empresa dá margem ao risco de que o fluxo de informações seja desordenado. Por exemplo, com a utilização da segunda forma de planejamento da implantação, a estrutura de dados dos sistemas pode não ser detalhada, possibilitando que algum dado necessário ao sistema de PFP e disponível no sistema de MRP não seja utilizado.

Este tipo pode ser aplicado preferencialmente nas seguintes circunstâncias:

- empresas que já possuem uma integração entre seus sistemas de auxílio às funções de PCP, e

- empresas que estejam iniciando a integração do PCP com outras funções e possuam grande volume de informações para processamento.

Este tipo é sugerido para aplicação nessas circunstâncias devido à grande performance no processamento de informações e à falta de relacionamento com o modelo de empresa. A comunicação entre sistemas através de um SGBD - Sistema Gerenciador de Base de Dados - permite o processamento de um grande volume de informações.

\section{Célula 3.3}

Esta célula apresenta a utilização de uma forma avançada de planejamento da implantação com um nível satisfatório de integração entre sistemas para grande número de aplicações. O elevado nível de planejamento da implantação se sobrepõe a algumas das limitações apresentadas pelo nível de integração de sistemas.

Nesta célula, a integração MRP-PFP permite a troca de um grande volume de dados com rapidez.

A integração PFP-Coleta é satisfatória, e o planejamento da implantação proposto possibilita que as transferências de arquivos sejam realizadas com eficiência.

A aplicação deste tipo pode cobrir grande variedade de modelos de empresas, abrangendo desde empresas médias, que estejam iniciando a integração de suas funções, até grandes empresas e conglomerados que já possuam projetos anteriores de integração. 


\section{Célula 3.4}

Nesta célula, a integração MRP-PFP é sofisticada e abrangente, assim como a integração do sistema de PFP com a coleta de dados do chão-de-fábrica.

A aplicação deste tipo é ampla. Isso porque ele pode ser utilizado na maior parte das empresas, desde as médias, que estejam iniciando o seu processo de integração, até conglomerados que processem grande quantidade de informações.

A aplicação deste tipo pode suportar a integração de todas ou quase todas as funções de uma empresa de manufatura. Essas funções e a comunicação entre elas são representadas no modelo estrutural da empresa. A utilização de um SGBD permite o armazenamento de todas ou quase todas as informações de uma empresa. Existem sistemas comerciais que se propõem a efetuar esta tarefa, baseados em sistemas gerenciadores de bases de dados. A aplicação destes sistemas requer um cuidadoso projeto de implantação, devido à sua complexidade.

\section{Aplicação da Implantação Integrada de um Sistema de PFP na Fábrica Integrada Modelo}

$\mathrm{N}$ esta seção é descrita a aplicação da implantação integrada de um sistema de PFP. Essa implantação ocorreu em um ambiente simulado, chamado de Fábrica Integrada Modelo.

A Fábrica Integrada Modelo (FIM) é um ambiente produtivo simulado, desenvolvido no Departamento de Engenharia Mecânica da Escola de Engenharia de São Carlos da Universidade de São Paulo (EESC-USP).

O processo produtivo da FIM é caracterizado pela utilização de sistemas integrados para a realização de todas as tarefas da manufatura, desde o recebimento de pedidos até a entrega do produto final. Este processo é realizado inteiramente por sistemas, sem a utilização de documentos impressos, simulando um ambiente produtivo real.

A célula adotada para implantação integrada de um sistema de PFP na FIM foi a 3.3, que representa a terceira forma de planejamento da implantação e o nível 3 de integração de sistemas.

A implantação integrada de um sistema de PFP na FIM resultou em um cenário de apresentação. Os cenários da FIM são caracterizados pela integração de sistemas. Na apresentação desses cenários é escolhida uma configuração do produto fabricado, por um dos participantes. A partir disso, é simulado todo o processo de negócios de uma empresa, passando pelas diversas áreas de uma empresa de manufatura, até a entrega do produto final.

Neste cenário podem ser mostrados principalmente dois tópicos:

- Integração entre um sistema de PFP e um sistema de planejamento dos recursos de manufatura (MRP II) e

- Fluxo das informações do processo de PCP em um ambiente de manufatura.

\section{Conclusões}

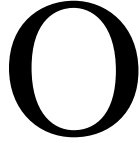

s sistemas de planejamento fino da produção são utilizáveis em diversos tipos de empresas. As características dessas empresas, suas necessidades e expectativas irão orientar a implantação integrada de um sistema de PFP.

A elaboração das matrizes de compatibilidade e adequação mostrou que as decisões e atividades relacionadas à implantação 
ficam facilitadas. Isto porque são caracterizadas as atividades relativas à implantação para as formas de planejamento da implantação e para os níveis de integração de sistemas. Assim, a partir da caracterização de uma empresa, é possível estruturar a implantação integrada de um sistema de PFP.

A utilização das matrizes de compatibilidade e adequação pode servir como um guia, para empresas de manufatura, na tomada de decisão quanto a promover uma implantação integrada de sistemas de PFP. Esse guia fornece uma orientação abrangente e que pode ser utilizada por empresas de portes variados e diferentes níveis de informatização.

A implantação integrada de um sistema de PFP na Fábrica Integrada Modelo (FIM) validou as propostas deste trabalho, mostrando que a sistemática proposta serve de orientação para empresas que necessitem realizar uma implantação integrada de um sistema de PFP.

\section{Referências Bibliográficas:}

BREMER, C.F.: Proposta de uma metodologia para o planejamento e implantação da manufatura integrada por computador, Tese de doutorado, EESC-USP, São Carlos, 1995.

MELLO, M.C.F.: Desenvolvimento de um sistema de programação visual e controle da produção assistido por computador, Tese de mestrado, EESC-USP, São Carlos, 1994.
ROZENFELD, H. \& FAVARETTO, F.: “Metodologia de implantação de um sistema de planejamento fino da produção”. Anais do Congresso Brasileiro de Engenharia Mecânica, Brasília, Brasil, 1993.

SCHEER, A.W.: CIM - Evoluindo para a fábrica do futuro. (tradução) Qualitymark, Rio de Janeiro, 1993.

\section{A SYSTEMATIC APPROACH FOR A LEITSTAND SOLUTION INTEGRATED IMPLANTATION}

\section{Abstract}

The changes in the world scenario are carrying the manufacturing enterprises towards a need in flexibility increase. The Production Planning and Control (PPC) function is one of the first areas that will be in position to have to this changes. The Leitstand systems are tools which purpose are to help the PPC to achieving this flexibility. The Leitstand systems make the link between the shop floor dynamics and the production planning environment, and its implantation is a critical factor. The Leitstand system needs to be integrated with other manufacturing supporting systems. The objective of this work is to propose a systematic approach to a Leitstand systems integrated implantation. This proposal was tried in a simulated environment, called Fábrica Integrada Modelo (FIM), and was tested and validated. 